\title{
Virus encoded circulatory miRNAs for early detection of prostate cancer
}

\author{
Jayoung Kim ${ }^{1,2,3^{*}}$, Seok Joong Yun ${ }^{4}$ and Wun-Jae Kim ${ }^{4 *}$
}

\begin{abstract}
Background: Prostate cancer (PCa) is the most commonly diagnosed cancer and kills about 28,000 American men annually. Although progress has been made in understanding the molecular features of different forms of the disease, PCa is considered incurable when it becomes resistant to standard therapies. Prostate specific antigen (PSA) test has been a gold standard of diagnosis for $\mathrm{PCa}$, however, it can result in lead to the unnecessary biopsies and treatment of indolent cancers due to the low specificity. Thus, the limitations of PSA screening for PCa have prompted much focus on strategies how to enhance the accuracy of PSA for distinction between aggressive and indolent cancers.

Discussion: Studies of miRNAs in PCa patients have suggested differentially expressed miRNAs between healthy controls and those with PCa, providing potential biomarker candidates using body fluids including urine and blood. Virus infection has been considered to associate with PCa incidence. Virus infected PCa cells may shed extracellular vesicles and communicate with neighboring cells, which were not infected yet, however, no mechanistic approaches were performed to understand the biology. The miRNAs composition in the shedding extracellular vesicles, and its role in PCa are completely undefined. In the near future, new insights to connect between the viral derived miRNAs and PCa progression might provide an opportunity to diagnose, risk prediction and therapeutic strategies.

Summary: The goal of this debate article is to provide a short review on miRNAs, virus infection and viral encoded miRNAs in PCa, with a primary focus on circulating miRNAs as potential non-invasive biomarkers for PCa patients.
\end{abstract}

Keywords: miRNAs, Virus, Extracellular vesicles, Prostate cancer

\section{Background}

Prostate cancer $(\mathrm{PCa})$ remains one of the most commonly diagnosed malignant tumor in men and the second leading cause of death from cancer [1-3]. Several new drugs including Radium-223, Cabazitaxel, Sipuleucel-T, Abiraterone, and Enzalutamide have shown significantly improved survival in castration-resistant metastatic disease (CRPC) patients in Phase 3 trials [4-8]. For early detection of PCa, urologists rely on serum prostate-specific antigen (PSA) testing or digital rectal examination (DRE) [9]. Serum PSA testing has successfully achieved a dramatic increase of $\mathrm{PCa}$ detection, however, PSA testing has a low specificity because an increase of PSA level is not a PCa-specific event.

\footnotetext{
* Correspondence: Jayoung.Kim@cshs.org; wjkim@chungbuk.ac.kr ${ }^{1}$ Departments of Surgery and Biomedical Sciences, Cedars-Sinai Medical Center, 8700 Beverly Blvd., Davis Room 5071, Los Angeles, CA 90048, USA ${ }^{4}$ Department of Urology, Chungbuk National University College of Medicine, 62 Kaeshin-dong, Heungduk-gu, Cheongju 361-763, Republic of Korea
} Full list of author information is available at the end of the article
Serum PSA levels are also elevated in men with benign prostatic hyperplasia and prostatitis. Limitations of DRE for the early detection derived from its low accuracy and the dependence on highly trained clinicians. Such a lack of PCa-specific early detection tool leads to create unnecessary biopsies or severe treatments for indolent PCa [2]. Development of methods of prebiopsy risk stratification and more simple, noninvasive, sufficiently sensitive and specific tests for PCa diagnosis would allow the stratification of $\mathrm{PCa}$ patients who are at the very early stage of disease $[1,10]$. Current efforts to improve the accuracy of PSA and develop new biomarkers for PCa may hold the promise of improving the screening, diagnosis, and monitoring of prostate cancer. PCA3, a non-coding and large chain RNA that is significantly overexpressed in PCa compared to nontumorous prostate cells, was introduced as a biomarker for PCa to show high sensitivity ( $52 \%$ to $58 \%$ ) and specificity (72\% to $87 \%$ ) [11-16]. The US FDA approved PCA3 as a risk assessment tool for $\mathrm{PCa}$ to guide prostatic biopsy 
among men with negative previous prostate biopsies. Urinary PCA3 assay combined with TMPRSS2:ERG is reported to improve the diagnostic accuracy $[17,18]$. Recently reported studies suggested that these requirements could be fulfilled using the diagnostic approach based on analysis of urine, which has become the future for non-invasive biomarker testing $[10,19]$.

This brief debate article is to discuss ad seek to characterize the circulating microRNAs (miRNAs) in urine with regard to $\mathrm{PCa}$. We aim to provide our audience the current knowledge, in particular, focused on clinical implication as a liquid biopsy with a clinically satisfactory degree of sensitivity and specificity.

\section{Discussion}

\section{MicroRNAs in prostate cancer} What are microRNAs?

Small non-coding microRNA (miRNA) molecules are known as post-transcriptional regulators involved in the regulation of gene and protein expression by interfering in the post-transcriptional level, resulting in the degradation and translation inhibition of mRNAs. Approximately more than 2,000 mature human miRNAs have been reported so far. These miRNAs are short (19-24 nt) and low molecular weight RNAs. miRNAs are derived from hairpin-like precursor transcripts (pre-miRNAs) and taken out of nucleus by a mediator protein, exportin 5 . Pre-miRNA is then cleaved by Dicer (a ribonuclease III enzyme) to excise the mature miRNAs in the form of siRNA-like duplexes and asymmetrical assembling of the mature miRNA strands. Since miRNAs interact with multiple messenger RNAs by binding the pairing of bases of the mRNAs and repress target gene expression, and regulate mRNA cleavage and mRNA decay initiated by the miRNA-guided rapid deadenylation, miRNAs have a wide variety of functions contributing to various pathological conditions including prostate cancer [20].

\section{How miRNAs are connected with PCa development and progression cancer?}

Many of miRNAs play roles in cell proliferation and apoptosis processes, thus miRNA expression profiles can be considered as useful biomarkers monitoring many types of cancer progression and treatment responses [21]. Previous studies have suggested several miRNA biomarker candidates associated with PCa [22]. miR-548c-3p was found significantly overexpressed in CRPC. Expression levels of miR-548c-3p negatively correlated with recurrence-free survival. miR-375 was significantly downregulated in $83.5 \%$ of PC patients compared to BPH controls. Metastatic CRPC patients with chemotherapy-resistant had the higher miR-21 levels compared with those with hormone dependent primary PCa. A combined diagnostic testing of miR-21 and PSA could stratify patients effectively. miR-21 was introduced as one of miRNAs of a multiple diagnostic profile, which includes miR-21,miR-141, and miR-221. miR-141 was independently identified as a biomarker of CRPC. Another combination panel of PSA and expression levels of let-7c, miR-30c, miR-141, and miR375 was suggested as diagnostic biomarkers for $\mathrm{PCa}$ screening outperforming the PSA testing alone. Some PCa-specific miRNAs were identified from mouse PCa model. A set of 46 miRNAs in the serum of transgenic mice with advanced adenocarcinoma PCa were identified. Among those, miR-141, miR-298, and miR-375 were found to be elevated in the serum of metastatic CRPC patients. In particular, miR-141 and miR-375 showed a correlation with disease outcome. Diagrammatic representation of miRNAs associated with $\mathrm{PCa}$ is shown in Fig. 1.

\section{What can be the target genes or pathways of miRNAs in $\mathrm{PCa}$ ?}

In the accumulated literature the roles of miRNAs in the pathobiology of PCa were found in cell cycle, apoptosis, epithelial to mesenchymal transition (EMT) and

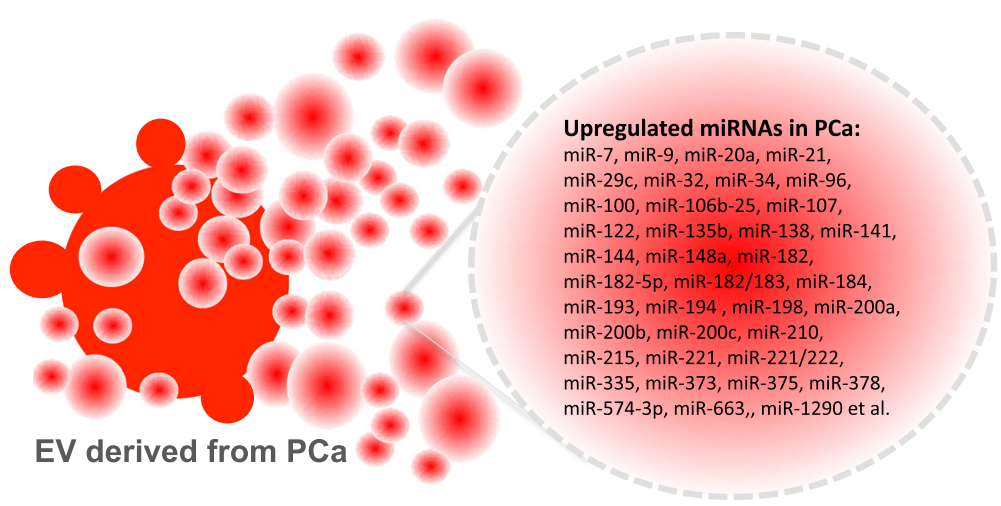

Fig. 1 miRNAs upregulated in PCa 
mesenchymal to epithelial transition (MET) states, invasion and metastasis, $\mathrm{PCa}$ stem cell, and androgen receptor (AR) pathway. PTEN-PI3 Kinase-AKT, EGFR and AR pathways are considered as the most important signaling pathways in PCa. Upregulation of miR-221/222 in PCa suggest a mechanistic link to $\mathrm{PCa}$ since miR-221/-222 plays a role in AR pathway regulation, ckit, PTEN, and TIMP3 et al. miR-124 was characterized to have a direct targeting of $\mathrm{AR}$ thereby inducing down-regulation of miR-125b and up-regulation of p53. When miR-125b is suppressed miR-125b effectors (p52, Puma, Bak1, and p14ARF) were downregulated. The regulation of miR-125b on apoptotic proteins (mitochondrial cytochrome C and Caspase-3) and NCOR2 (a corepressor of AR) was well known. Another PCaassociated miRNA, miR-let-7c regulates AR pathway and involves in the conversion of hormone dependent PCa to CRPC.

\section{Urinary microRNAs as prostate cancer biomarkers}

Several cancer-associated miRNAs were found in circulating body fluids such as urine, which make it possible to develop non-invasive biomarkers mainly due to their ease of access and stability. Only few studies for $\mathrm{PCa}$-associated miRNA in urine were reported. Five of the miRNAs that were differentially quantified in $\mathrm{PCa}$ patients compared to controls (miR-107, miR-574-3p, miR375, miR200b and miR141) were successfully quantified in urine of men with cancer, which were much higher in $\mathrm{PCa}$ patients than that of healthy volunteers. Such reports provide evidence that circulating miRNAs might be a next-generation biomarker and contribute to cancer screening in non-invasive liquid biopsy. Two additional promising miRNAs, miR-141 and miR-375 were found in the patient blood. In particular, data from this study showed that metastatic PCa patients have approximately 50 fold higher miR-141 levels, compared to the healthy individuals.

\section{Virus infection and prostate cancer}

Among the various viruses, herpes virus is one of the viruses most commonly related to carcinogenesis. Several epidemiological studies evaluated the association between herpes virus infection and prostate cancer risk, although results were inconsistent. Herpes virus plays an important role in the pathogenesis of cancer via the inhibition of cell apoptosis and stimulation of DNA synthesis, which may ultimately lead to PC. Previous meta-analysis indicated that infection by herpes simplex virus type $2(\mathrm{HSV}-2)$ or human herpesvirus 8 (HHV-8) may be associated with a higher prostate cancer risk. However, there has not been a study to elucidate the potential mechanism of HSV-2 infection underlying viral PC carcinogenesis. A potential link between HPV infection and PCa risk was attempted to study the causal role of HPV-16 in prostate carcinogens. A significant increase of $\mathrm{PCa}$ risk related with HPV-16 infection was observed. However, this is still contradictory since other epidemiological studies could not find same association [23-25]. Therefore, an elaborate and comprehensive demonstration of the association between herpes virus infection and prostate cancer risk is of significance [24]. Further investigations and large-sample studies are required to and the relationship between herpes virus infection and prostate cancer risk.

Our recent findings demonstrated the overexpression of herpes virus-encoded miRNAs in urine samples from prostate cancer patients, compared to those of control subjects [19]. Interestingly, hsv1-miR-H18 and hsv2miR-H9-5p detected in urine samples showed better diagnostic performance than tPSA levels in patients within the PSA grey zone. A recent meta-analysis showed that HSV infection is associated with an increased $\mathrm{PCa}$ risk, however, it remains puzzling how these two particular vir-miRNAs contribute to $\mathrm{PCa}$. To better understand the biological contribution of hsv1miR-H18 and hsv2-miR-H9-5p to PCa, the geographic distribution, gender difference and socioeconomic variation of virus infection and their possible impact on prostate cancer should be considered to investigate (Fig. 2).

\section{Viral miRNAs circulating through biofluids}

Viruses also encode their own sets of miRNAs, which they use to control the expression of either the host's genes and/or their own. In the past few years' evidence of the presence of cellular miRNAs in extracellular human body fluids such as serum, plasma, saliva, and urine has been accumulated [26, 27]. Furthermore, it has been also demonstrated that miRNAs secreted by virusinfected cells are transferred to and act in uninfected recipient cells [19].

The first viral-encoded miRNAs was found in cells infected with EBV [27, 28]. The majority of natural viruses found to encode miRNAs. In DNA viruses (such as herpes virus, polyomavirus, ascovirus, baculovirus, iridovirus, and adenovirus families), a DNA component to replication cycle can exploit the initiating host miRNA biogenesis machinery in the nucleus, where they replicate and cause long-term persistent infections. Among DNA viruses, which account for the majority of known virus-encoded miRNAs, $95 \%$ of viral miRNAs known today are of herpes virus origin. However, little has been identified about the role of circulating miRNAs from the virus-infected cells. 


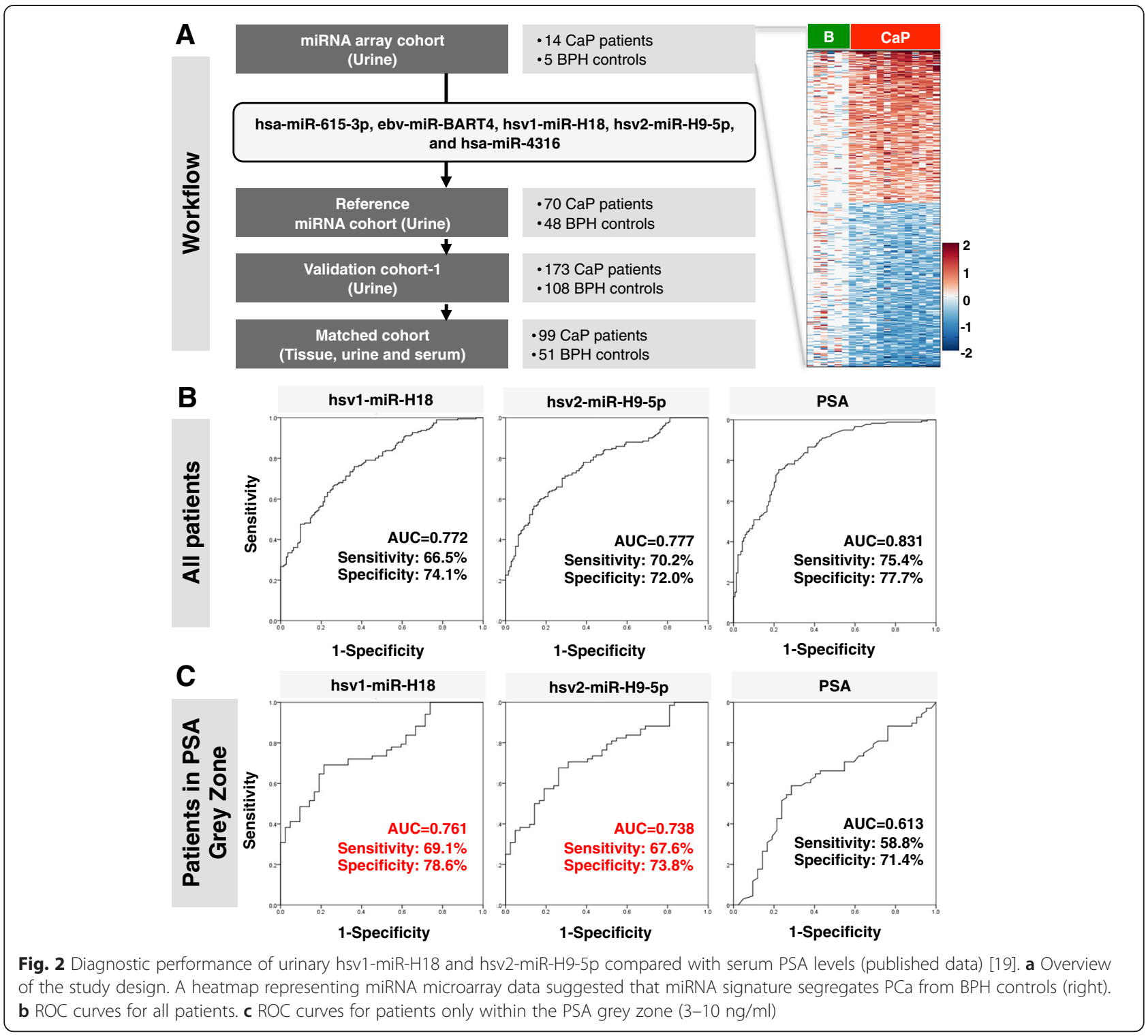

\section{Extracellular vesicles and their biological role of $\mathrm{EV}$ in prostate cancer}

Cells can release different types of vesicles, which have been difficult to categorize in a definitive manner. The transmission of vesicles from cancer cells to other cell types has been the subject of intensive studies in recent years. It is a process that can make it possible a sophisticated form of cellular communication through the delivery of highly complex and dynamic cargo. As a delivery vehicle between cells, extracellular vesicles (EV) have been considered as a molecular cargo mediating a communication between cells in the microenvironment. Although EV were originally considered to be a means for exclusion of garbage molecules from cells, it is now clear that EV alter signaling pathways and have a biological influence in neighboring cells [29].
Also, since EV have the bioactivity of their molecular cargo and can be readily isolated from multiple biological fluids (e.g., urine, serum, plasma, pleural effusion, saliva), EV has been considered as a non-invasive biomarker candidate [30-32].

There are multiple types of EV, the small sealed membrane vesicles that are produced from cells differing in size, biology, contents, and secretory mechanisms. Exosomes and microparticles (MPs) are two distinct groups of EV. Exosomes are originated from the inward budding of the limiting membrane of multivesicular bodies (MVBs), while MPs are in bigger size vesicles formed by the outward membrane budding. Vesicles that are produced from cells during cell death are apoptotic bodies.

It is known that PCa patients had higher levels of urinary exosomes than the healthy donors. The membranes 
of exosomes are resistant to the osmeolytic and proteolytic activity of urine, indicating that exosomes are quite stable in urine. PC cell-derived EV can deliver various genetic factors such as nucleic acids, including DNA, mRNA, miRNA, and small non-coding RNAs, as well as oncoproteins and metabolites, leading to the horizontal transfer of oncogenic information to neighboring immune cells, vessel cells, or cancer cells [33, 34]. EVmediated RNA transfer provides benefits as a communicasome [35-37]. However, little is known how EV-mediated transfer of these molecules enters into recipient cells, and how specific miRNA species can be sorted into EV. Our series of publications in this field suggest that EV shedding can influence on microenvironment of PCa by modulating immune cell proliferation and activation [38].

\section{EV influence immune response in prostate cancer}

Role of immune response caused/mediated by miRNA was suggested by a series of studies showing that EV-derived miRNAs participate in the regulation of inflammatory responses. A recent report from our group demonstrated that EV derived from amoeboid phenotype of prostate cell may influence the immune response of the tumor microenvironment. A tritiated thymidine ( ${ }^{3} \mathrm{H}$-thymidine) incorporation assay revealed that EV contain miRNAs (e.g., miR-125a), which are transferred to tumor microenvironment leading to proliferative inhibition of immune cells. Other interesting findings suggest immune response may be mediated by miRNAs regulating Toll-like receptors (TLRs), which play an important role in immune response and inflammation including tissue repair and tissue injury-induced inflammation. In PCa cell lines, several miRNAs (e.g., has-miR-29b, $-29 c,-148 b$, and -152 ) were upregulated by TLR3 activation, leading to antitumoral effects on PCa. EV derived from dendritic cells contain miR-155 (a promoter of inflammatory responses) and miR-146a (a mediator of immune suppression), which are known as important players to regulate inflammation, alter the gene expression of inflammation related targets and reprogramm the response to endotoxin [39, 40].

\section{EV released from virus-infected cells}

In virology field, biology of extracellular vesicles are well understood compared to cancer mainly because virus should be enveloped to be released from infected host. The accumulating evidence suggests that viruses, such as retroviruses, hepatitis $C$ virus, herpes simplex virus, Epstein-Barr virus, Coxsackie virus B3 (CVB3), utilize the cellular vesiculation pathway for budding and assembly, immune evasion, and intercellular communication. It is also well known that many virally infected cells secrete vesicles (most of cases, exosomes) containing various viral proteins and genetic materials such as RNAs. Studies on herpes simplex virus-infected cells demonstrated that virus communicate via vesicles. In the case of EpsteinBarr virus, recent reports suggested that herpes virus utilizes exosomes, one kind of extracellular vesicles, as a mechanism of cell-to-cell communication and transfers signaling competent proteins and functional miRNAs to uninfected neighbor cells. For example, exosomes released from infected cells have been shown to contain coreceptors for HIV, which can enhance virus entry into cells. Proteins in the secreted exosomes from virusinfected cells can induce apoptosis in CD4 T cells, and contribute innate immune response.

\section{$\mathrm{EV}$ as cargoes to deliver viral miRNAs}

EV-mediated communication would allow the virus to respond to the cellular microenvironment. Virus-infected cells continuously shed and transfer EV to uninfected neighboring cells. Throughout EV shedding and secretion to extracellular space, the virus-encoded miRNAs were delivered to other cells, leading to alteration of miRNAmediated gene repression and intercellular communication. Thus, the presence of viral-encoded miRNAs in EV suggests that virus-infected cells perturb gene expression in the surrounding tissue, resulting in destruction of the immune system. Exosomes secreted from HIV-infected alveolar macrophages have been identified to carry viral miRNA (vir-miRNA) such as vmiR88, vmiR99, and vmiRTAR. It has not been clearly identified how vir-miRNA composition is decided and recruited to EV. Vir-miRNAs might have a role in cancer initiation by blocking of major tumor suppressors (e.g., p53) or acceleration of cancer development by evading cellular immune response (e.g., mi17-92).

These findings still raise a number of exciting questions. Does virus-infected prostate cells secrete miRNAs using EV as a cargo? What are the target genes of the abundantly secreted viral miRNAs? Are extracellular vesicles shed only in the absence of viral replication? Is there any evidence for functional miRNA delivery in vivo? If so, what's the biological mechanism? In addition, the detail mechanism is currently lacking whether virus infection promotes miRNAs sorting into $\mathrm{EV}$, or whether this secretion is a selective and specific process.

\section{Conclusions}

In PCa biomarker development, the greatest unmet need remains: a biomarker that stratifies men at risk of aggressive $\mathrm{PCa}$ or a biomarker that identify the early stage of patients who need active surveillance, eventually leading to a reduction of unnecessary interventions. Although miRNAs might have many useful clinical applications for patients with $\mathrm{PCa}$, many additional studies are warranted to clarify their function and regulation during tumorigenesis and tumor progression. The 
studies to determine the role of circulating miRNAs during $\mathrm{PCa}$ progression would have the potential that $\mathrm{PCa}$ patients can be molecularly stratified based on their miRNAs profile in urine samples. In addition, the studies could uncover important clues about underlying disease mechanisms.

Urine analyses have great potential to be adapted in clinical practice, based on its non-invasiveness. Identification of non-invasive clinical indicators of $\mathrm{PCa}$ would be one of the most important advances achievable in this field. Our current mechanistic understanding of shedding vesicle biology and function, especially in the context of virus infection and communication with host, is not mature enough. Identification of potential candidates of the circulating miRNAs signature in patient urine samples would have significant implications for an alternative and/or supportive diagnostic tool for PCa.

Unnecessary diagnostic procedures would be minimized for patients with early stage of $\mathrm{PCa}$, contributing to the easier diagnostic assessment and to the reducing associated public health and economic burden to patients. In addition, the studies to understand role and biology of the circulating miRNAs have significant clinical relevance to public health since it will improve accuracy of predicting patient survival, identifying responsiveness candidates of PCa patients. Furthermore, we envision that this study will also provide the mechanistic data to address the long-term goal of the PCa field identifying new treatments tailoring more specific and effective therapies for $\mathrm{PCa}$, thus it holds great promise for the treatment of high risk PCa patients.

\section{Abbreviations}

PCa: Prostate cancer; CRPC: Castration-resistant metastatic disease; PSA: Prostate-specific antigen; DRE: Digital rectal examination; miRNAs: MicroRNAs; EMT: Mesenchymal transition; MET: Mesenchymal to epithelial transition; AR: Androgen receptor; HSV-2: Herpes simplex virus type 2; HHV-8: Human herpesvirus 8; EV: Extracellular vesicles; MPs: Microparticles; MVBs: Multivesicular bodies; CVB3: Coxsackie virus B3.
\end{abstract}

\section{Competing interests}

The authors have stated that they have no conflicts of interest.

\begin{abstract}
Authors' contributions
$J K, S Y$ and WK participated in the design of the study and performed the analysis of references. JK and WK supervised study design and led obtaining funding. JK drafted the manuscript, and SY and WK participated critical revision of the manuscript. All authors read and approved the final manuscript.
\end{abstract}

\footnotetext{
Acknowledgements

This work was supported by the National Research Foundation of Korea (NRF) grant funded by the Korea government (MSIP) (No. NRF2014R1A2A1A09006983), the Ministry of Education, Science and Technology (2012R1A1A4A01008753 and 2013R1A1A2004740) (to W-J.K), NIH grants 1R01DK100974-01, DoD W81XWH-15-1-0415, U24 DK097154, NIH NCATS UCLA CTSI UL1TR000124, IMAGINE NO IC Research Grant, the Steven Spielberg Discovery Fund in Prostate Cancer Research Career Development Award, Interstitial Cystitis Association (ICA) Pilot Grant, and a Fishbein Family IC Research Grant by ICA, New York Academy of Medicine; Boston Children's Hospital Faculty Development (to J.K.).
}

\section{Author details}

'Departments of Surgery and Biomedical Sciences, Cedars-Sinai Medical Center, 8700 Beverly Blvd., Davis Room 5071, Los Angeles, CA 90048, USA. ${ }^{2}$ Departments of Medicine, University of California, Los Angeles, CA, USA. ${ }^{3}$ Department of Surgery, Harvard Medical School, Boston, MA, USA. ${ }^{4}$ Department of Urology, Chungbuk National University College of Medicine, 62 Kaeshin-dong, Heungduk-gu, Cheongju 361-763, Republic of Korea.

Received: 4 August 2015 Accepted: 18 November 2015

Published online: 26 November 2015

\section{References}

1. Attard G, Parker C, Eeles RA, Schroder F, Tomlins SA, Tannock I, et al. Prostate cancer. Lancet. 2015.

2. Behesnilian AS, Reiter RE. Risk stratification of prostate cancer in the modern era. Curr Opin Urol. 2015;25(3):246-51.

3. Siegel RL, Miller KD, Jemal A. Cancer statistics, 2015. CA Cancer J Clin. 2015;65(1):5-29.

4. Hoy SM. Abiraterone acetate: a review of its use in patients with metastatic castration-resistant prostate cancer. Drugs. 2013;73(18):2077-91.

5. McGann S, Horton ER. Radium-223 dichloride: a novel treatment option for castration-resistant prostate cancer patients with symptomatic bone metastases. Ann Pharmacother. 2015;49(4):469-76.

6. Mukherji D, Omlin A, Pezaro C, Shamseddine A, de Bono J. Metastatic castrationresistant prostate cancer (CRPC): preclinical and clinical evidence for the sequential use of novel therapeutics. Cancer Metastasis Rev. 2014;33(2-3):555-66.

7. Vuong W, Sartor O, Pal SK. Radium-223 in metastatic castration resistant prostate cancer. Asian J Androl. 2014;16(3):348-53.

8. Zustovich F, Fabiani F. Therapeutic opportunities for castration-resistant prostate cancer patients with bone metastases. Crit Rev Oncol Hematol. 2014;91(2):197-209.

9. Smith RA, Manassaram-Baptiste D, Brooks D, Doroshenk M, Fedewa S, Saslow D, et al. Cancer screening in the United States, 2015: a review of current American cancer society guidelines and current issues in cancer screening. CA Cancer J Clin. 2015;65(1):30-54.

10. Trock BJ. Circulating biomarkers for discriminating indolent from aggressive disease in prostate cancer active surveillance. Curr Opin Urol. 2014;24(3):293-302.

11. Birnbaum JK, Feng Z, Gulati R, Fan J, Lotan Y, Wei JT, et al. Projecting benefits and harms of novel cancer screening biomarkers: a study of PCA3 and prostate cancer. Cancer Epidemiol Biomarkers Prev. 2015;24(4):677-82.

12. Day JR, Jost M, Reynolds MA, Groskopf J, Rittenhouse H. PCA3: from basic molecular science to the clinical lab. Cancer Lett. 2011;301(1):1-6.

13. Hessels D, Klein Gunnewiek JM, van Oort I, Karthaus HF, van Leenders GJ, van Balken B, et al. DD3(PCA3)-based molecular urine analysis for the diagnosis of prostate cancer. Eur Urol. 2003;44(1):8-15. discussion 15-16.

14. Loeb S. Prostate cancer: Predicting prostate biopsy results-PCA3 versus phi. Nat Rev Urol. 2015;12(3):130-1.

15. Marks LS, Fradet Y, Deras IL, Blase A, Mathis J, Aubin SM, et al. PCA3 molecular urine assay for prostate cancer in men undergoing repeat biopsy. Urology. 2007;69(3):532-5.

16. Wei JT, Feng Z, Partin AW, Brown E, Thompson I, Sokoll L, et al. Can urinary PCA3 supplement PSA in the early detection of prostate cancer? J Clin Oncol. 2014;32(36):4066-72

17. Sidaway P. Prostate cancer: Urinary PCA3 and TMPRSS2:ERG reduce the need for repeat biopsy. Nat Rev Urol. 2015.

18. Tomlins SA, Day JR, Lonigro RJ, Hovelson DH, Siddiqui J, Kunju LP, et al. Urine TMPRSS2:ERG Plus PCA3 for Individualized Prostate Cancer Risk Assessment. Eur Urol. 2015

19. Yun SJ, Jeong P, Kang HW, Kim YH, Kim EA, Yan C, et al. Urinary MicroRNAs of prostate cancer: virus-encoded hsv1-miRH18 and hsv2-miR-H9-5p could Be valuable diagnostic markers. Int Neurourol J. 2015;19(2):74-84.

20. Ayub SG, Kaul D, Ayub T. Microdissecting the role of microRNAs in the pathogenesis of prostate cancer. Cancer Genet. 2015

21. Lin XJ, Chong Y, Guo ZW, Xie C, Yang XJ, Zhang Q, et al. A serum microRNA classifier for early detection of hepatocellular carcinoma: a multicentre, retrospective, longitudinal biomarker identification study with a nested case-control study. Lancet Oncol. 2015;16(7):804-15.

22. Korzeniewski N, Tosev G, Pahernik S, Hadaschik B, Hohenfellner M, Duensing S. Identification of cell-free microRNAs in the urine of patients with prostate cancer. Urol Oncol. 2015;33(1):16 e17-22. 
23. Heidegger I, Borena W, Pichler R. The Role of Human Papilloma Virus in Urological Malignancies. Anticancer Res. 2015;35(5):2513-9.

24. Ge X, Wang $X$, Shen P. Herpes simplex virus type 2 or human herpesvirus 8 infection and prostate cancer risk: A meta-analysis. Biomed Rep. 2013;1(3):433-9.

25. Caini S, Gandini S, Dudas M, Bremer V, Severi E, Gherasim A. Sexually transmitted infections and prostate cancer risk: a systematic review and meta-analysis. Cancer Epidemiol. 2014;38(4):329-38.

26. Meckes Jr DG, Shair KH, Marquitz AR, Kung CP, Edwards RH, Raab-Traub N. Human tumor virus utilizes exosomes for intercellular communication. Proc Natl Acad Sci U S A. 2010;107(47):20370-5.

27. Qi P, Han JX, Lu YQ, Wang C, Bu FF. Virus-encoded microRNAs: future therapeutic targets? Cell Mol Immunol. 2006;3(6):411-9.

28. Qureshi A, Thakur N, Monga I, Thakur A, Kumar M. VIRmiRNA: a comprehensive resource for experimentally validated viral miRNAs and their targets. Database (Oxford). 2014;2014.

29. Minciacchi VR, Freeman MR, Di Vizio D. Extracellular vesicles in cancer: exosomes, microvesicles and the emerging role of large oncosomes. Semin Cell Dev Biol. 2015:40:41-51.

30. Hessels D, Schalken JA. Urinary biomarkers for prostate cancer: a review. Asian J Androl. 2013;15(3):333-9.

31. Webber J, Yeung $\vee$, Clayton A. Extracellular vesicles as modulators of the cancer microenvironment. Semin Cell Dev Biol. 2015;40:27-34.

32. Kim J, Morley S, Le M, Bedoret D, Umetsu DT, Di Vizio D, et al. Enhanced shedding of extracellular vesicles from amoeboid prostate cancer cells: potential effects on the tumor microenvironment. Cancer Biol Ther. 2014;15(4):409-18.

33. Hessvik NP, Sandvig K, Llorente A. Exosomal miRNAs as Biomarkers for Prostate Cancer. Front Genet. 2013;4:36.

34. Greening DW, Gopal SK, Mathias RA, Liu L, Sheng J, Zhu HJ, et al. Emerging roles of exosomes during epithelial-mesenchymal transition and cancer progression. Semin Cell Dev Biol. 2015;40:60-71.

35. Tetta C, Ghigo E, Silengo L, Deregibus MC, Camussi G. Extracellular vesicles as an emerging mechanism of cell-to-cell communication. Endocrine. 2013:44(1):11-9.

36. Yanez-Mo M, Siljander PR, Andreu Z, Zavec AB, Borras FE, Buzas El, et al. Biological properties of extracellular vesicles and their physiological functions. Journal of extracellular vesicles. 2015;4:27066.

37. Yoon YJ, Kim OY, Gho YS. Extracellular vesicles as emerging intercellular communicasomes. BMB Rep. 2014;47(10):531-9.

38. Choi DY, You S, Jung JH, Lee JC, Rho JK, Lee KY, et al. Extracellular vesicles shed from gefitinib-resistant nonsmall cell lung cancer regulate the tumor microenvironment. Proteomics. 2014;14(16):1845-56.

39. Alexander M, Hu R, Runtsch MC, Kagele DA, Mosbruger TL, Tolmachova T, et al. Exosome-delivered microRNAs modulate the inflammatory response to endotoxin. Nat Commun. 2015;6:7321

40. Galli R, Paone A, Fabbri M, Zanesi N, Calore F, Cascione L, et al. Toll-like receptor 3 (TLR3) activation induces microRNA-dependent reexpression of functional RARbeta and tumor regression. Proc Natl Acad Sci U S A. 2013:110(24):9812-7.

\section{Submit your next manuscript to BioMed Central and we will help you at every step:}

- We accept pre-submission inquiries

- Our selector tool helps you to find the most relevant journal

- We provide round the clock customer support

- Convenient online submission

- Thorough peer review

- Inclusion in PubMed and all major indexing services

- Maximum visibility for your research 